\section{Exfoliation syndrome in the Reykjavik eye study}

Arnarsson et al examined the age- and gender-specific prevalent and 5-year incident risk of developing exfoliation syndrome (XFS) in a population-based random sample of citizens 50 years and older. One thousand forty-five persons had baseline examination in 1996; 846 of the 958 survivors (88.2\%) had a follow-up examination in 2001. Age, female gender, increased iris pigmentation, moderate use of alcohol and self-reported asthma were found to correlate significantly with prevalence risk of XFS at baseline. They also observed that food items that are possibly surrogates for antioxidative effect correlated with decreased risk of XFS.

See page 831.

\section{Ethnic differences in optic nerve head and RNFL parameters in children}

Samarawickrama et al examined ethnic differences in optic nerve head and RNFL parameters between European Caucasian and East Asian children aged 6-12 years. Of 4118 children examined in the Sydney Childhood Eye Study, 3382 (82.1\%) had OCT (Zeiss Stratus, Fast optic disc and RNFL scans) data suitable for analysis. After adjusting for age, gender, axial length, birth weight and optic-disc area, East Asian children had similar mean vertical disc diameters to European Caucasians but 30-43\% larger mean vertical cup diameters, resulting in larger mean cup/disc ratios. East Asian children had thicker mean average RNFL $(105.45 \mu \mathrm{m}$ and $107.92 \mu \mathrm{m})$. These anatomical variations could contribute to better understanding of apparent racial differences in glaucoma susceptibility. See page 871.

\section{TGFBI mutational analysis in inherited corneal dystrophy}

Vincent et al characterised the molecular basis for corneal dystrophy in 19 unrelated individuals affected with granular, fleck, lattice, and posterior polymorphous dystrophy. All five patients with granular dystrophy had R555W mutation, and H626P was identified in a pedigree with intermediate dystrophy of Bowman layer. No other mutations were detected including stromal dystrophy cases, suggesting greater genetic heterogeneity than is currently known in this group of disorders. See page $\mathbf{8 3 6}$

Refractive error, axial length, and ACD in British adults: the EPICNorfolk Eye Study

Foster et al describe the distribution and demographic and socioeconomic correlates of refractive error and related ocular biometry in an older British population (2519 people). Among phakic individuals, axial length of the eye was strongly inversely correlated with refractive error in men and women. Anterior chamber depth varied with age and sex, but not with socioeconomic status. Axial length of the eye was strongly, independently related to height, weight and social class. Educational status was the strongest determinant of axial length. See page 827 .

\section{Descemet membrane endothelial} keratoplasty with a stromal rim Studeny et al describe a novel technique for preparation and transplantation of posterior corneal lamellae consisting of endothelium and bare Descemet membrane with a stromal supporting rim (DMEK-S) using the big bubble technique. The retrospective case series that underwent DMEK-S comprised 20 eyes of 18 patients with endothelial dysfunction. Best-corrected visual acuity (BCVA) and endothelial cell density (ECD) were measured preoperatively and 12-24 months after DMEK-S. Primary graft failure occurred in two eyes. The average ECD at 1 year was 1608 ( \pm 503 ) cells/ $\mathrm{mm}^{2}$. The loss of donor cornea, during preparation, decreased to $5 \%$ as more experience was acquired. As this approach is entirely manual, it has the potential to become widely adopted. See page 909.
Bruch's membrane and choroidal macrophages in AMD

Cherepanoff et al determined the submacular Bruch's membrane (BrM) macrophage count and the choroidal and BrM macrophage immunophenotype in 125 human eyes (normal, normal aged, early $\mathrm{AMD}$ and, geographical atrophy). CD68 and inducible nitric oxide synthase (iNOS) immunohistochemistry was performed on 16 human eyes (normal, early AMD, geographical atrophy, and disciform scarring). They observed that CD68+ macrophages were found in the choroid of normal human eyes but they did not express iNOS. Expression of iNOS by choroidal macrophages was associated with early $\mathrm{AMD}$ and active disciform scarring. iNOS expression was absent in BrM macrophages, suggesting immunomodulatory differences between the choroid and BrM macrophages. See page 918

\section{PDT and intravitreal bevacizumab} for myopic choroidal

\section{neovascularisation}

Baba et al compared the long-term outcome with photodynamic therapy (PDT) and intravitreal bevacizumab (IVB) for myopic choroidal neovascularisation (mCNV) in 24 eyes divided into Group A (12 eyes treated by PDT) and Group B (12 eyes treated by $1.25 \mathrm{mg}$ IVB). The age and best-corrected visual acuity (BCVA) were matched between the two groups. The BCVA did not change after PDT but was significantly improved from $0.75 \pm 0.25$ to $0.50 \pm 0.38 \log$ MAR units at 24 months after IVB. The CFT was significantly reduced in both groups at 24 months. The size of the chorioretinal atrophy surrounding the CNV (CRA) was larger in group $A$ than in group $B$ at 24 months. The CRA size was correlated with the BCVA. The authors conclude that IVB is more effective than PDT in treating $\mathrm{mCNV}$. Incomplete visual recovery after $\mathrm{PDT}$ might be related to enlargement of the CRA after PDT. See page 864 . 